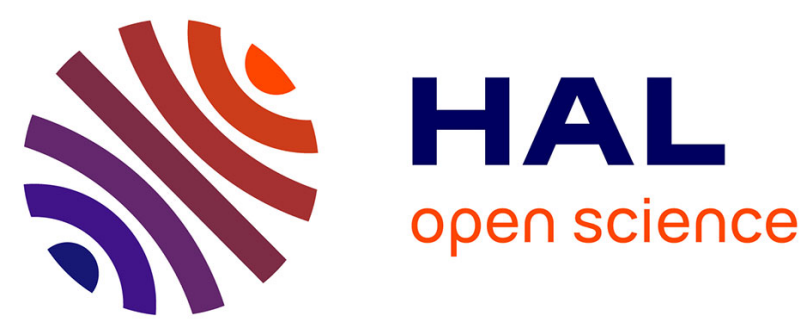

\title{
Heterogeneous Microscopic Dynamics of Intruded Water in a Superhydrophobic Nanoconfinement: Neutron Scattering and Molecular Modeling
}

J Wolanin, L Michel, D Tabacchioni, J M Zanotti, J Peters, I Imaz, B Coasne, M Plazanet, Cyril Picard

\section{- To cite this version:}

J Wolanin, L Michel, D Tabacchioni, J M Zanotti, J Peters, et al.. Heterogeneous Microscopic Dynamics of Intruded Water in a Superhydrophobic Nanoconfinement: Neutron Scattering and Molecular Modeling. Journal of Physical Chemistry B, 2021, 125 (36), pp.10392-10399. hal-03332577

\section{HAL Id: hal-03332577 \\ https://hal.science/hal-03332577}

Submitted on 2 Sep 2021

HAL is a multi-disciplinary open access archive for the deposit and dissemination of scientific research documents, whether they are published or not. The documents may come from teaching and research institutions in France or abroad, or from public or private research centers.
L'archive ouverte pluridisciplinaire HAL, est destinée au dépôt et à la diffusion de documents scientifiques de niveau recherche, publiés ou non, émanant des établissements d'enseignement et de recherche français ou étrangers, des laboratoires publics ou privés. 


\title{
Heterogeneous Microscopic Dynamics of Intruded Water in a Superhydrophobic Nanoconfinement: Neutron Scattering and Molecular Modeling
}

\author{
J. Wolanin, ${ }^{\dagger}$ L. Michel, ${ }^{\dagger}$ D. Tabacchioni, ${ }^{\dagger}$ J. M. Zanotti, ${ }^{\ddagger}$ J. Peters, ${ }^{+, \mathbb{I}}$ I. Imaz, ${ }^{\S}$ B. Coasne, ${ }^{\dagger}$ M. Plazanet,,${ }^{*}+$ \\ and C. Picard*,+ \\ +Univ. Grenoble Alpes, CNRS, LIPhy, 38000 Grenoble, France \\ $\ddagger$ Laboratoire Lon Brillouin, CEA, CNRS, Universit Paris-Saclay, CEA Saclay, 91191 Gif-sur-Yvette Cedex, France \\ IIInstitut Laue Langevin, 38042 Grenoble, France \\ $\S$ Catalan Insitute of Nanoscience and Nanotechnology, Barcelona, Spain
}

E-mail: marie.plazanet@univ-grenoble-alpes.fr; cyril.picard@univ-grenoble-alpes.fr

\begin{abstract}
With their strong confining porosity and versatile surface chemistry, Zeolitic Imidazolate Frameworks - including the prototypical ZIF-8 - display exceptional properties for various applications. In particular, the forced intrusion of water at high pressure $(\sim 25 \mathrm{MPa})$ into ZIF-8 nanopores is of interest for energy storage. Such a system reveals also ideal to study experimentally water dynamics and thermodynamics in ultra-hydrophobic confinement. Here, we report on neutron scattering experiments to probe the molecular dynamics of water within ZIF-8 nanopores under high pressure up to $38 \mathrm{MPa}$. In addition to an overall confinement-induced slowing down, we provide evidence for strong dynamical heterogeneities with different underlying molecular dynamics. Using complementary molecular simulations, these heterogeneities are found to correspond to different microscopic mechanisms inherent to vicinal molecules located in strongly adsorbing sites (ligands) and other molecules nanoconfined in the cavity center. These findings unveil a complex microscopic dynamics, which results from the combination of surface residence times and exchanges between the cavity surface and center.
\end{abstract}

\section{Introduction}

Interfacial and confined water has become a scientific field in itself at the crossroad of physics, chemistry and biology with significant implication for applied science such as in geophysics, mechanical/fluid engineering, etc. ${ }^{1,2}$ Such water, which displays marked differences with its bulk counterpart, is of importance for situations where physicochemical interactions with surfaces are at play: protein folding, biological nanochannels, nanofiltration/phase separation, catalysis, etc. ${ }^{1,3,4}$ Despite the microscopic origin and nanometric range of these physicochemical interactions, the impact of interfaces on the water structure and dynamics - with a pronounced dependence on confinement size, geometry and chemistry - manifests itself at the macroscopic scale as illustrated by considering water flow properties. ${ }^{5-8}$ Hydrophilic, i.e. wetting, surfaces are typically characterized by a no slip boundary condition while hydrophobic, i.e. non-wetting, surfaces are known to exhibit slippage. ${ }^{9,10}$ Such slip boundary conditions are broadly considered as a mean to overcome the permeability-selectivity trade-off in membrane science such as for medical applications, water desalination, blue en- ergy harvesting ${ }^{11}$ (with the goal to compete with high performance biological selective nanochannels ${ }^{3}$ ).

Despite their significant impact at the macroscopic level, the molecular specificities of water near solid surface remain only partly elucidated. At the microscopic level, a large body of experimental and numerical studies is available on the dynamics of water confined within wetting, i.e. hydrophilic, nanoporous media. In this case, the strong molecular interactions with the hydrophilic surfaces combined with severe in-pore confinement cause a decrease in water translational diffusion coefficient - i.e. a slowing down of water dynamics which gets more pronounced with tighter confinement and/or stronger surface interactions. ${ }^{12-16}$ In partially wetting materials, available data on moderately hydrophobic materials such as carbon nanotubes suggest a more complex scenario with strongly enhanced water transport as probed using permeability measurements. ${ }^{17}$ While such faster transport can be rationalized by elucidating the very specific interactions between water and the host surface (weakened hydrogen-bond network, curvature-induced friction decrease, etc.), ${ }^{18}$ it does not necessarily imply an increase in the diffusion coefficient and associated faster selfdynamics. As a matter of fact, even if the solid surface impact is less pronounced with hydrophobic surfaces, a decreased self-diffusion coefficient with respect to that of bulk water has been also reported in the literature by different groups. ${ }^{13,19}$ Nevertheless, in all these studies dedicated to hydrophilic and so-called hydrophobic materials, the affinity of water for the solid matrices is always strong enough to promote a spontaneous water condensation within the pores at a bulk vapor pressure lower than the saturation pressure.

Beyond hydrophilic and moderately hydrophobic materials, the microscopic dynamics in superhydrophobic nanoporous solids - such as all-silica zeolites and some Metal Organic Frameworks for which a pressure much larger than the water saturation pressure is needed to force water into the porosity - remains to be elucidated. Despite a well-established non-wetting thermodynamic behavior, ${ }^{20}$ the molecular mechanisms ruling water transport in these materials involve complex phenomena which are often overlooked in such superhydrophobic environment (residence steps, in-pore relocation, etc.). In particular, the elemental dynamical mechanisms remain almost unexplored using microscopic experiments (e.g. neutron scattering, nuclear magnetic resonance). In this context, Zeolitic Imidazolate Frameworks (ZIF) are attractive candidates owing to their tunable 
chemistry, variety of topology and nanoscale pore size. ${ }^{21-24}$ ZIF-8 is made of tetrahedral zinc ions linked by imidazolate organic groups (see insert in Figure 1). These two different moieties form a cubic structure of spherical cages, $11.6 \AA$ in diameter, interconnected by $3.4 \AA$ narrow gates which fill with water at a pressure of the order of $25 \mathrm{MPa}$ at room temperature. The ZIF-8 structure, which is superhydrophobic, presents local heterogeneities as regularly encountered in biological confinement. The large microporous volume of ZIF-8 $\left(\sim 0.4 \mathrm{~cm}^{3} / \mathrm{g}\right)^{25}$ is experimentally of interest to reach - after intrusion - a significant content of confined water with respect to bulk water. While water within ZIF-8 has been largely explored from a structural viewpoint, ${ }^{22,26-29}$ its dynamics has received only very little attention. On the one hand, Han et al. ${ }^{30}$ considered the impact of defects on the dynamics of gas water molecules in ZIF-8, on the other hand Fraux et $a l .{ }^{28}$ reported a ten-fold decrease in liquid water self diffusivity. Despite these pioneering works, we note that the details of the molecular mechanisms leading to such a dynamics slowing down remain to be identified.

Here, we report on a coupled experimental and molecular simulation investigation of the microscopic dynamics of intruded water in superhydrophobic confinement. To the best of our knowledge, we performed the first direct measurement of the diffusion coefficient of liquid water in such superhydrophobic environment by means of quasi-elastic neutron scattering (QENS). Our experiments are combined with a dual Grand Canonical Monte Carlo/molecular dynamics simulation strategy which sheds light on the heterogeneous dynamics of water confined in such hydrophobic environment. While we confirm the average slowing down of the dynamics for confined water, careful analysis of our experimental data in the light of molecular simulations unveils a complex microscopic dynamics related to two different dynamical mechanisms: reduced dynamics for water located close to strongly adsorbing sites (ligands) and bulk-like water in the cavity center.

\section{Material and Methods}

The molecular simulation approach first involves Grand Canonical Monte Carlo simulations to determine the number of adsorbed water molecules as a function of pressure. Then starting from configurations obtained at a pressure where the ZIF-8 porosity is filled with water, we performed molecular dynamics simulations to compare bulk and confined water dynamics. A supercell consisting of a $2 \times 2 \times 2$ ZIF- 8 unit cell (the cubic lattice parameter is approximately $1.7 \mathrm{~nm}$ ) was built to conduct these calculations. Periodic boundary conditions were used along the 3 space directions to avoid finite size effects. Following previous works on water in ZIF, the TIP4P ( Transferable Intermolecular Potential-4 Point) allatom model is used to describe the water molecule while both a nominal and rescaled force field proposed by Amrouche et al. ${ }^{31}$ is used to model the water/ZIF-8 molecular interactions. All details on the molecular simulation strategy can be found in the Supporting Information (S5).

QENS experiments were performed at Institut LaueLangevin (ILL, Grenoble) on the time-of-flight spectrometer IN6-Sharp with an incident wavelength of $5.1 \AA$ - leading to an elastic energy resolution of $70 \mu \mathrm{eV}$ (dynamics faster than $\sim 10 \mathrm{ps)}$ over the momentum transfer $q$-range of $0.4-2$ $\AA^{-1}$. Because of the large hydrogen incoherent cross section for neutrons, the signal arising from hydrogen motions of water dominates the scattered intensity, therefore providing a measurement of the self-dynamics of water in the sample. Descriptions of the experimental environment together with calibration and adequate background measurements are given in the Supporting Information (S2). In brief, an optimized aluminium high pressure cell was designed ${ }^{32}$ to prepare a sub-millimetric uniform and dense layer of ZIF-8 powder (BASF, Basolite ${ }^{\circledR}$ Z1200), with similar volume fractions in intra-grain pores and inter-grain bulk voids. Measurements were performed under hydrostatic pressures of 4, 20, 28 and $38 \mathrm{MPa}$ which are below and above the water intrusion pressure of $25 \mathrm{MPa}$ (see Figure 2a) at ambient temperature. ${ }^{24}$ The two lower pressures correspond to a matrix empty of liquid whereas the two higher pressures correspond to a matrix filled with liquid of interest for the study of water dynamics in this superhydrophobic framework. The reproducibility of the measurements was assessed by performing several short measurements during successive intrusion cycle from low to high pressure.

\section{Results and Discussion}

We first investigate the water dynamics, using molecular simulation, from the mean square displacements (MSD) for bulk and confined water with respect to time (Figure 1). In the long time limit $t>1 \mathrm{~ns}$, the short-wave vector displacements (large distances) are dominated by translational motion where both the $\mathrm{O}$ and $\mathrm{H}$ atoms diffuse over the same distance on a given time scale. While the Fickian regime is reached on a short time scale for bulk water $(t \sim 100 \mathrm{ps})$, we found that much longer simulations are required to investigate this normal diffusion regime for confined water (typically, $t \sim 10 \mathrm{~ns}$ ). The diffusivity of water confined in ZIF8 at $300 \mathrm{~K}$ is found to be smaller than its bulk counterpart; typically, $D_{T} \sim 3.4 \times 10^{-9} \mathrm{~m}^{2} / \mathrm{s}$ for bulk water while $D_{T}$ $\sim 2.6 \times 10^{-10} \mathrm{~m}^{2} / \mathrm{s}$ for confined water. These two values obtained with the TIP4P water model being 30\% larger than the ones obtained with the SPC/E model (extended simple point charge model) ${ }^{28}$. However, importantly, the ten-fold decrease in the water self diffusivity between bulk and con-

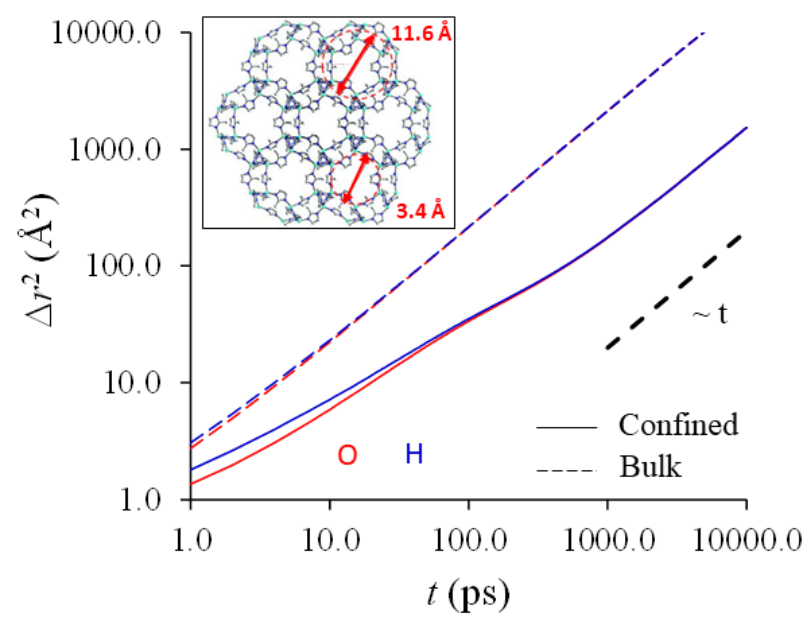

Figure 1: Mean square displacements $\Delta r^{2}$ as obtained using molecular dynamics for the $\mathrm{O}$ and $\mathrm{H}$ atoms of water in bulk water (dashed lines) and confined in ZIF-8 (solid lines). The black dashed line is a guide to the eye to indicate the Fickian regime where $\Delta r^{2} \sim t$. The insert shows the ZIF-8 structure. 
fined values is independent of the force field or its scaling (as detailed in the Supplementary Information).

In the short time limit $(t<10-100 \mathrm{ps})$, for both bulk and confined water, the $\mathrm{O}$ and $\mathrm{H}$ mean square displacements depart from each other due to rotational effects. Interestingly, while the mean square displacements for bulk water is timescale independent in the sense that it exhibits a single regime once rotational effects are no longer noticeable, the data for confined water indicate a heterogeneous dynamics with different regimes. In more detail, between the time range dominated by rotation/translation coupling $(t<100$ ps) and the normal Fickian regime ( $t \sim 1-10$ ns), a subdiffusive regime where $\Delta r^{2} \sim t^{\alpha}$ with $\alpha<1$ is observed.

At this stage it is useful to comfort these initial numerical results with experimental values of the diffusion coefficient $D_{T}$. The confined water dynamics is extracted from the dynamical structure factor $S(q, \omega) .{ }^{33}$ The experimental $S(\omega)$ spectra (summed over the whole $q$-range) at the different investigated hydrostatic pressures are shown in Figure $2 \mathrm{~b}$. The contribution from the ZIF-dry matrix, which is purely elastic, confirms the absence of QENS signal that could arise from imidazole ring rotation on the investigated time scale. Moreover, complementary measurements have been performed on the spectrometer IN13 (ILL), covering a shorter $(\sim 100 \mathrm{ps})$ timescale than IN6-SHARP. The results show that the rotational motion of the 2-methyl-imidazole rings is not influenced by the intrusion process (as it occurs on a timescale that is included in the elastic line of the present experiment). Details are presented in the Supporting Information (S1). We also observe that spectra below $P_{\text {int }}=25 \mathrm{MPa}$ (4 and $20 \mathrm{MPa}$ ) are very similar as well as those above $P_{\text {int }}(28$ and $38 \mathrm{MPa}$ ). Below $25 \mathrm{MPa}$, only inter-grain water is present outside the ZIF-8 grains as the nanopores are empty. Thus, the observed intensity increase between 20 and $28 \mathrm{MPa}$ (Figure 2c) provides evidence for the presence of water within the nanopores above $P_{i n t}$. The obtained high-pressure sig- nal is therefore a direct measurement of the water dynamics in such superhydrophobic confinement. The limited ZIF-8 compressibility, ${ }^{34}$ which leads to minor deformations of the matrix on the considered pressure range - essentially related to a slight dilation of the order of $0.5 \%$ occurring within the narrow pressure window associated to the filling process ${ }^{35}$ _ is expected to not alter the liquid water dynamics within the pores after intrusion. The superimposition of signals measured at $28 \mathrm{MPa}$ and $38 \mathrm{MPa}$ confirms that once the matrix is filled, the pressure increase does not alter the water dynamics, neither directly through a potential change of water structure nor indirectly because of the flexibility of the matrix. The dynamical structure factor, $S(q, \omega)$, in the investigated energy range, can be approximated by the product of the Debye - Waller factor, accounting for the vibrational motions, with the decoupled diffusive motions, such as rotational, $S_{R}(q, \omega)$, and translational, $S_{T}(q, \omega)$, ones: ${ }^{33}$

$$
S(q, \omega)=\left[S_{T}(q, \omega)+S_{R}(q, \omega)\right] \exp \left(-\frac{q^{2}\left\langle u^{2}\right\rangle}{3}\right)
$$

with $\left\langle u^{2}\right\rangle$ the mean-square vibrational amplitude of the hydrogen atoms. In a first approach, both translational and rotational motions were described using a Lorentzian function of unconstrained half-width at half-maximum (hwhm), $\Gamma$. $\Gamma_{T}$ and $\Gamma_{R}$ refer to the translational and rotational motions, respectively. In the Fickian regime, the variation of $\Gamma_{T}$ is linear with $q^{2}$ as $\Gamma_{T}=D_{T_{\text {Fick }}} q^{2}$ where $D_{T_{\text {Fick }}}$ is the translational self-diffusion coefficient. For the localized rotational motion, the width is $q$-independent: $\Gamma_{R}=2 D_{R}$ with $D_{R}$ the rotational diffusion constant. Such a phenomenological model relying on two Lorentzian functions provides a reliable description of the water dynamics under different thermodynamical conditions. We emphasize that the appropriate background for each pressure condition is subtracted in order to isolate successively the inter-grain and intra-grain wa-
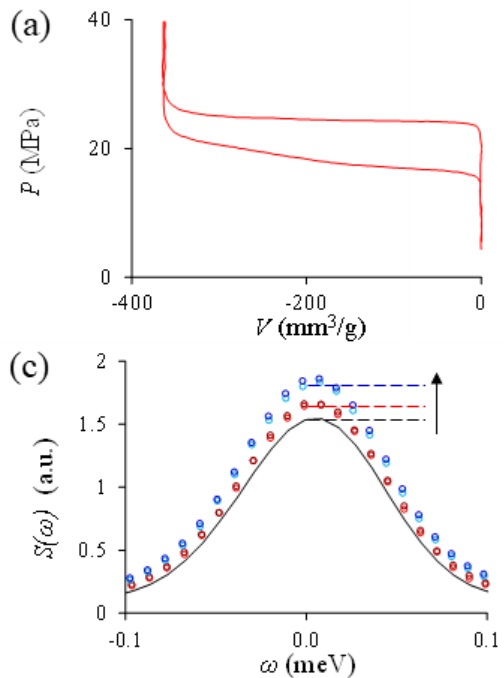
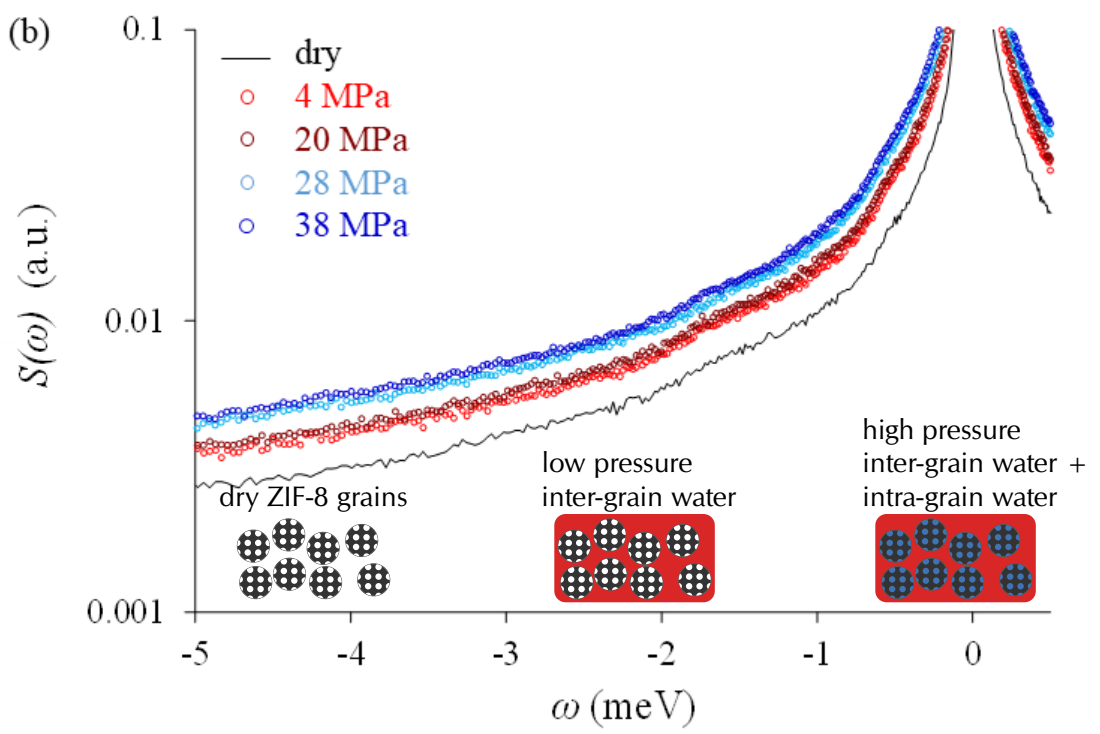

Figure 2: (a) Experimental water intrusion/extrusion cycle at room temperature in a ZIF-8 material. $V(P)$ indicates the intruded and extruded volume $V$ of water per g of ZIF material as a function of the exerted pressure $P$. (b) $S(\omega)$ summed over $q$ [from 0.4 to $2 \AA^{-1}$ ] of the quasi-elastic spectra $S(q, \omega)$ obtained for dry ZIF-8 (black), low-pressure water in contact with ZIF-8 (red) and high-pressure water intruded in ZIF-8 (blue). Inset: schematics of these three situations. (c) Zoom on the elastic peak showing the overall increase of the intensity upon water addition. 


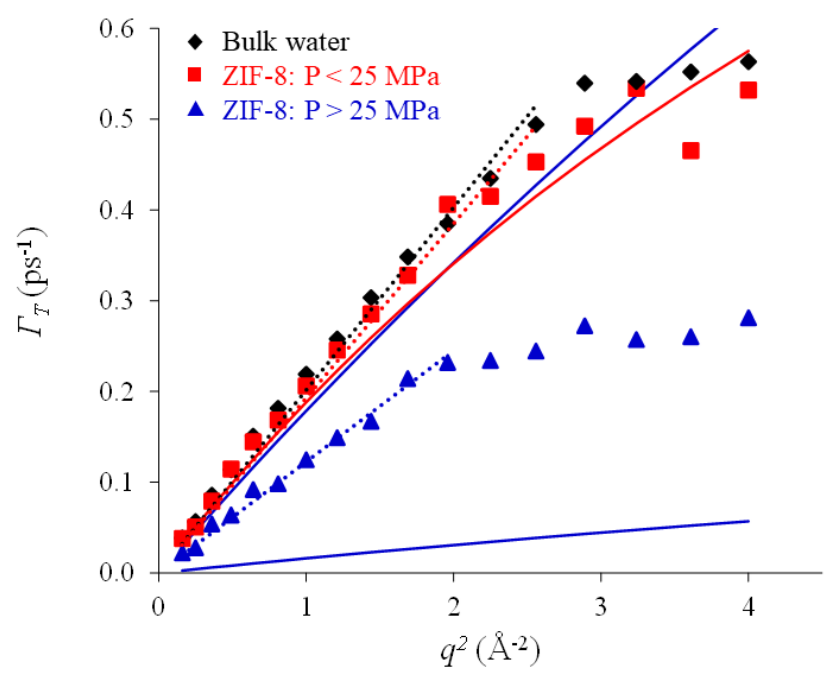

Figure 3: Half-width at half-maximum $\Gamma_{T}$ of the translational contribution versus $q^{2}$ for bulk water (black), low-pressure water in contact with ZIF-8 (red) and high-pressure water intruded in ZIF-8 (blue). Error bars are smaller than the symbol size. The symbols refer to the results obtained with the analysis 1 (Eq. 1). The dashed segments indicate the Fickian regime where $\Gamma_{T} \sim q^{2}$. The solid lines are attributed to the results obtained with the analysis 2 (Eq. 2) using the jump diffusion model. We note the presence of two solid blue lines associated to the heterogeneous behavior of the intruded highpressure water obtained with the analysis 2 .

The translational dynamics for bulk water and inter-grain water obey to Fick's law for $q^{2}<3 \AA^{-2}$. The corresponding self-diffusion coefficients, of $2.01 \times 10^{-9} \mathrm{~m}^{2} / \mathrm{s}$ and $1.92 \times$ $10^{-9} \mathrm{~m}^{2} / \mathrm{s}$ respectively, are very close to each other, and in very good agreement with values found in the literature for bulk water at $295 \mathrm{~K}^{37}$ (incidentally we note that the MSD value measured with the numerical TIP4P water model is $70 \%$ larger than these experimental values). The water surrounding the grains is therefore only poorly influenced by the inter-grain boundaries on the time and space scales investigated. The deviation from Fick's law at large $q$ is expected because of the simplified model - single Lorentzian - used for the rotational dynamics (instead of the full derivation taking into account higher Lorentzian orders and coupling between the two relaxation dynamics - see Eq. 3 and in S3 for more details). ulation, to a linear Fickian regime that extends much beyond $q^{2} \sim 2 \AA^{-2}$. As detailed in the Supporting Information (S3), the deviation from Fick's law beyond this $q$ value, marked by the onset of a plateau for $\Gamma_{T}$, provides a signature of the non homogeneous behavior of intra-grain water, due to the influence of the discrete atomic structure surrounding water (also highlighted in Figure 1 with the subdiffusive regime at short times). To further analyze this departure from an effective average behavior, a more rigorous model is needed to cover and accurately describe both the Fickian and non-Fickian dynamical regimes.

Table 1: Numerical values of the extracted fitting parameters for the analysis $1 . D_{T_{\text {Fick }}}$ was calculated with the Fick's law on the appropriate $q$-range.

\begin{tabular}{|c|c|c|}
\hline Analysis 1: eq. 1 & $D_{T_{\text {Fick }}} \times 10^{-5}\left[\mathrm{~cm}^{2} / \mathrm{s}\right]$ & $D_{R}\left[\mathrm{ps}^{-1}\right]$ \\
\hline Bulk water & $2.01 \pm 0.03$ & $2.06 \pm 0.01$ \\
ZIF-8: $\mathrm{P}<25 \mathrm{MPa}$ & $1.92 \pm 0.04$ & $2.09 \pm 0.02$ \\
ZIF-8: $\mathrm{P}>25 \mathrm{MPa}$ & $1.22 \pm 0.02$ & $1.75 \pm 0.02$ \\
\hline
\end{tabular}

We performed therefore a deeper analysis based on a formal description of water translation through the use of the jump diffusion model ${ }^{33}$ coupled to isotropic rotational diffusion in a sphere of radius $R$ (equal to the $\mathrm{OH}$ distance in a water molecule $\sim 1 \AA$ ). Each population will then be described by the new fitting model:

$$
S(q, \omega)=\exp \left(-\frac{q^{2}\left\langle u^{2}\right\rangle}{3}\right) S(q, \omega)_{T \otimes R}
$$

with

$$
\begin{aligned}
S(q, \omega)_{T \otimes R}= & \left(\frac{1}{\pi} \frac{\Gamma_{T}}{\omega^{2}+\Gamma_{T}^{2}}\right) \otimes \\
& \left(A_{0}(q) \cdot \delta(\omega)+\sum_{i=1}^{\infty} A_{i}(q) \cdot\left(\frac{1}{\pi} \frac{\Gamma_{i}}{\omega^{2}+\Gamma_{i}^{2}}\right)\right)
\end{aligned}
$$

where $\Gamma_{T}$ is defined within the jump-diffusion model:

In contrast to inter-grain water, the translational dynamics for intra-grain water follows Fick's law up to $q^{2} \sim 2 \AA^{-2}$ only. In the low $q$ Fickian regime, the confinement effect leads to a decrease of both translational and rotational diffusion coefficients with respect to their bulk counterpart. The slowing down measured experimentally is only of the order of $30 \%$ with respect to the bulk values. Such a limited departure from the bulk dynamics should lead, for a single water pop-

$$
\Gamma_{T}=\left(\frac{D_{T_{\text {Jump }} q^{2}}}{1+\tau D_{T_{\text {Jump }} q^{2}}}\right)
$$

with $\tau$ the residence time, $A_{i}(q)=(2 i+1) j_{i}^{2}(q R)$ where $j_{i}$ is the spherical Bessel function of order $i$ and $\Gamma_{i}=i(i+1) D_{R}$.

As expected, a single water population only is not sufficient to accurately fit our experimental data on the entire $(q, w)$ range. On the other hand, a good fit is obtained using two water populations (ratio of 70/30 for fast and slow population, respectively). Each of these two populations are described with the model given in Eqs. 2 and 3 (fitting curves are presented in the Supporting Information (S2)). The resulting fitting parameters are shown in Table 2. The fast population is similar to bulk water while the slow population has a translational diffusion coefficient one order of magnitude lower as inferred from the MSD obtained using molecular simulation (Figure 1). As detailed in the Supporting Information (S3), at low $q$, the average dynamics of the two populations is similar to the one presented in Analysis 1 with an effective translational diffusion coefficient $\sim 30 \%$ than that of bulk water. This moderate deviation at low $q$ is related to the small proportion of the slow water population with respect to the fast one. At high $q$, however, the slow water popula- 
Table 2: Numerical values of the extracted fitting parameters of the confined water in ZIF-8: P>25 MPa for the Analysis 2

\begin{tabular}{|c|c|c|c|}
\hline Analysis 2: eq. 2 & $D_{T_{\text {Jump }} \times 10^{-5}\left[\mathrm{~cm}^{2} / \mathrm{s}\right]}$ & $\tau[\mathrm{ps}]$ & $D_{R}\left[\mathrm{ps}^{-1}\right]$ \\
\hline ZIF-8: P $<25 \mathrm{MPa}$ & $2.09 \pm 0.05$ & $0.55 \pm 0.01$ & $1.83 \pm 0.01$ \\
\hline \hline ZIF-8: P $>25 \mathrm{MPa}$ : Population 1 & $1.87 \pm 0.02$ & $0.25 \pm 0.05$ & $2.18 \pm 0.04$ \\
\hline ZIF-8: P $>25 \mathrm{MPa}$ Population 2 & $0.167 \pm 0.007$ & $2.55 \pm 1.6$ & $0.17 \pm 0.01$ \\
\hline
\end{tabular}

tion, despite its limited proportion, becomes a leading term which contributes to the plateau observed for $\Gamma_{T}$ in Figure 3 in the framework of analysis 1 . Furthermore, moving now to the temporal domain using the inverse time Fourier transform of the formal model Eq. 2 (see Supporting Information S4) for the two populations, the translational term expresses as the sum of two time-decaying exponentials. At long time this translational term should be controlled by the slower exponential decay only associated to the slow population. This means that at long time a Fickian regime with a diffusion coefficient of the order of $1.7 \times 10^{-10} \mathrm{~m}^{2} / \mathrm{s}$ is expected. This analysis of experimental data confirms, at long time, the tenfold decrease observed from numerical MSD and reveals, at short time, that the heterogeneous dynamics of confined water in ZIF-8 can be interpreted as the interplay between a slow and a main bulk-like water population.

Attempting the same two-population decomposition using numerical data, a similar trend - even if to a less extent - is also observed when focusing on short time scales. As shown in Figure 4a, the self (incoherent) intermediate scattering function, $f(q, t)$ (inverse time Fourier transform of $S(q, \omega)$ ) extracted from molecular dynamics simulations for bulk water conforms to the classical behavior expected for Fickian diffusion. The superimposition of the curves plotted with respect to $q^{2} t$ reveals that the rotational contribution is restricted to a very short time scale. This is further confirmed by the large value of $D_{R} \sim 1 \mathrm{ps}^{-1}$ obtained from the fit of $f$ - inset of Figure $4 \mathrm{a}$ - using the inverse time Fourier transform of the formal model Eq. 2 (see Supporting Information S4). The dominant translational contribution of the form $\exp \left(-D_{T_{\text {Iump }}} q^{2} t\right)$ is characterized by a diffusion coefficient $D_{T_{\text {Iump }}}$ which agrees with the one inferred from MSD calculations. In contrast, as previously observed from our experimental data and molecular simulation $\operatorname{MSD}, f(q, t)$ for confined water display a non-Fickian behavior which can be approximated again with the contribution of a fast and a slow population (ratio 60/40) - each of them modeled with the inverse time Fourier transform of Eq. 2 (see inset of Figure 4a). Nevertheless, $D_{T_{\text {Ium }}}$ for the fast population remains four times smaller than that for bulk water; this is a

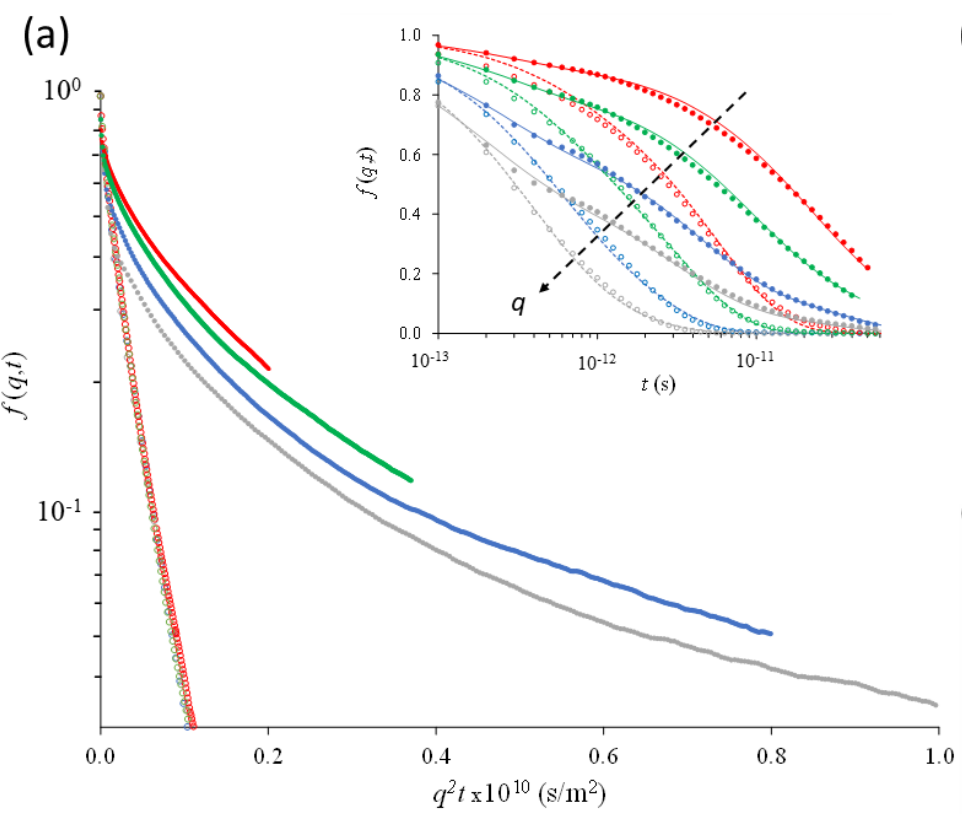

(b)

(c)
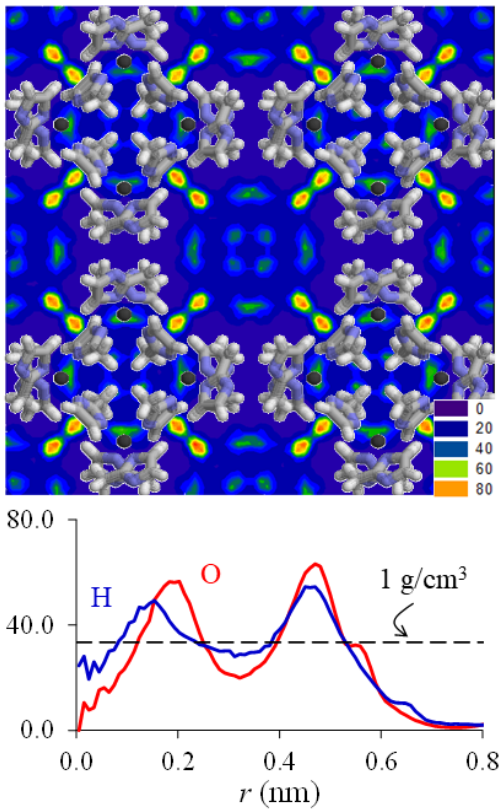

Figure 4: (a) Self (incoherent) intermediate scattering function $f(q, t)$ as a function of $q^{2} t$ for different scattering vector $\mathrm{q}=\mid$ $\mathbf{q} \mid$ for bulk water (open circles) and for water confined in ZIF-8 (full dots). The color code is as follows: $q=0.7 \AA^{-1}$ (red), $q$ $=1.0 \AA^{-1}$ (green), $q=1.5 \AA^{-1}$ (blue), and $q=2.0 \AA^{-1}$ (grey). Inset: $f(q, t)$ according to $t$ for bulk (open circle) and confined water (full dots). Dashed lines, bulk water: fit with a single water population using the inverse Fourier transform of Eq. 2 with $D_{T_{\text {Jump }}}=4.2 \times 10^{-9} \mathrm{~m}^{2} / \mathrm{s}, \tau=0.9 \mathrm{ps}$ and $D_{R}=0.8 \mathrm{ps}^{-1}$. Full lines, confined water: fit with a fast and a slow population (ratio $60 / 40$ ) with $D_{T_{\text {Iump }}}=1.1 \times 10^{-9} / 3.4 \times 10^{-10} \mathrm{~m}^{2} / \mathrm{s}, \tau=0.25 / 20 \mathrm{ps}$ and $D_{R}=2 / 0.1 \mathrm{ps}^{-1}$ (b) Contour plot showing the density of water in a ZIF-8 material as obtained using Grand Canonical Monte Carlo simulations. The density increases with color as follows: blue, green, yellow, orange and red. (c) Simulated density profiles $\rho(r)$ for the $\mathrm{O}$ and $\mathrm{H}$ atoms of water intruded in ZIF-8. $r$ is the distance from the center of the ZIF cages shown in the contour plot in (b). The dashed line indicates the bulk density of water at room temperature.) 
major difference with respect to experimental results. The rotational diffusion coefficient of the slower population being 20 times smaller than the fast population, in qualitative agreement with experimental results, the rotational contribution extends to much longer time than for bulk water and contributes to the separation of the curves observed in Figure $4 \mathrm{a}$ at large $q^{2} t$ values. Interestingly, the functions $f$ do not present a linear asymptotic trend at long $q^{2} t$ values that would be expected in the presence of two distinct water populations only. This means that such a basic yet efficient decomposition to quantitatively analyze experimental and numerical data does not fully reflect the heterogeneity of water dynamics in such complex materials partly controlled by residence times at surfaces (see Bousige et al. ${ }^{38}$ for a recent discussion on intermittent diffusion in complex nanoporous media).

At the microscopic level, the strong variations of water density as obtained from GCMC simulation (see Figure $4 \mathrm{~b}$ and Figure 4c) reveal and confirm a complex heterogeneous water structure within the ZIF-8 porosity. These data, which are consistent with previous molecular simulation in the field, ${ }^{22}$ show that water is adsorbed on specific sites, close to the ligands at a distance about $0.4-0.5 \mathrm{~nm}$ from the pore center [see marked density spots in Figure 4b], and in less adsorbing sites located closer to the pore center [see less marked density spots at a distance $\sim 0.2 \mathrm{~nm}]$. Although these adsorbing sites are hydrophobic enough to not trap water at ambient pressure, their relative affinity for the intruded water contribute to the marked slowing down its dynamics observed at long time.

For times much longer than the residence time, exchanges between the different adsorbing sites contribute to the complex heterogeneous dynamics and hence to the effective behavior accessible experimentally. Such a contribution from this molecular intermittence can be investigated by means of the diffusion propagator $G(r, t)$ (inverse space and time Fourier transform of $S(q, \omega))$ which corresponds to the probability that a molecule gets located at a position $r$ at a time $t$ while being at $r=0$ at a time $t=0$. At short times $(t<10 \mathrm{ps})$, $G(r, t)$ shown in Figure 5a for both $\mathrm{O}$ and $\mathrm{H}$ atoms of confined water is monomodal and reflects local displacements $(r \sim 0.1 \mathrm{~nm})$ which are similar to those found for bulk water on the same time scale (although of a slightly smaller amplitude in confinement). For larger times $(t>10 \mathrm{ps})$, the propagator becomes bimodal for $\mathrm{O}$ and $\mathrm{H}$ atoms, therefore indicating two characteristic translational modes coexisting on the same time scale. We show in the insert of Figure 5a the propagators for the different water populations confined in ZIF-8 (note the use of a log scale for improved visualization purpose). In more detail, we measured the propagator for molecules initially located at time $t=0$ in the strongly adsorbing sites close to the ZIF-8 ligands (see density contour plot shown in Figure $4 \mathrm{~b}$ ) and that for molecules initially located in the cavity center not directly in contact with ZIF-8 ligand/surface. Such data confirm that the fast diffusion mode (i.e. subpeak in the propagator located at distance $r \sim 3.5 \AA$ ) corresponds to molecules located within the cavity center while the slow diffusion model corresponds to molecules initially located in the strongly adsorbing sites. Interestingly, for both contributions, some molecules undergo displacements corresponding to the other mode. This is due to the fact that, during the characteristic time $t=10 \mathrm{ps}$ considered here, some molecules leave the cavity center to migrate towards the strongly adsorbing sites and reciprocally.

Additionally to the analysis of the translational motions,
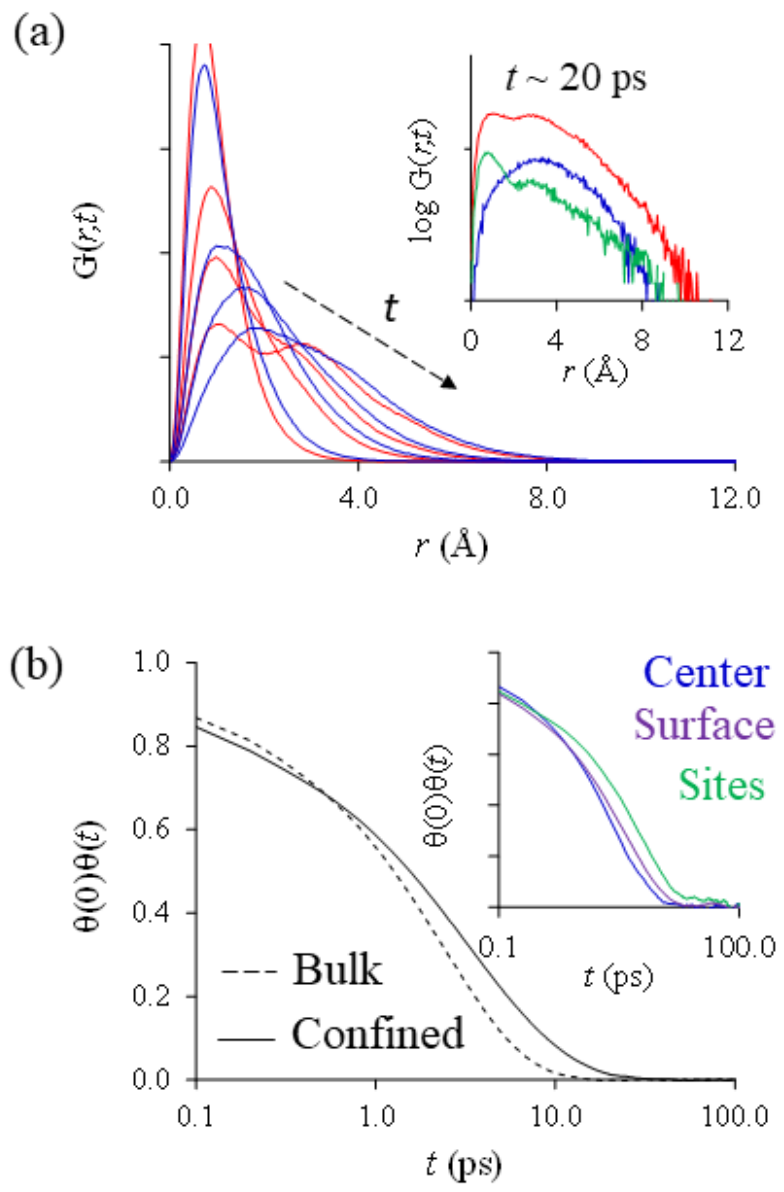

Figure 5: (a) Diffusion propagator $G(r, t)$ as obtained using molecular dynamics at room temperature for the $\mathrm{O}$ (red lines) and $\mathrm{H}$ (blue lines) atoms of water confined in ZIF-8. The dashed line is a guide to the eye to indicate that the data are estimated at increasing times $t=1,5,10$ and $20 \mathrm{ps}$. As discussed in the main text, the insert shows the different contributions to the $G(r, t)$ data for the $\mathrm{O}$ atoms at $t=$ 20 ps: all molecules in the ZIF-8 cage (red), molecules in the cage center (blue) and molecules in strongly adsorbing sites (green). (b) Rotational autocorrelation time $\theta(t) \theta(0)$ showing the change in a water molecule time at a time $t=0$ and a time $t$ later. The dashed and solid lines are for bulk water and water confined at room temperature in ZIF-8. The insert shows the same data for confined water but for different subpopulations: molecules in the cage center (blue), molecules at the cage surface (purple), and molecules in strongly adsorbing sites (green).

we extracted the rotational relaxation time distribution for water in the different regions of the ZIF- 8 cavities to clarify more specifically the impact of the surface heterogeneities ${ }^{39}$ (see Figure $5 b$ ). The timescale of rotational motions enables indeed a more local analysis since molecules, over such a short timescale, do not significantly exchange between different sites. Owing to their intrinsically slow dynamics, the rotational motion of the molecules located in the strongly adsorbing sites is slower than for molecules in the pore center again with a typical rotational relaxation time a few times larger than for water in the pore center. Such a slow rotational motion only applies to molecules in the strong adsorbing sites. For molecules at the ZIF-8 cavity surface, away 
Acknowledgement The authors thank the Institut LaueLangevin (Grenoble, France) for the allocation of neutron beam time. The neutron data set is available at DOI: http://10.5291/ILL-DATA.EASY-537. This work was supported by the French Research Agency (ANR LyStEn 15CE06-0006, ANR TWIST 17-CE08-0003). Some of the computations in this paper were performed using the Froggy platform of the GRICAD infrastructure (https: / / gricad.univgrenoble-alpes.fr), which is supported by the Rhne-Alpes region (GRANT CPER07-13 CIRA) and the Equip@Meso project (reference ANR-10-EQPX-29-01) of the programme Investissements dAvenir supervised by the French Research Agency. We thank Claude Payre and Jérôme Giraud for high pressure equipment and the design of high pressure cell. We are grateful to David Farruseng who initiated fruitful collaboration for this work.
(2) Kavokine, N.; Netz, R. R.; Bocquet, L. Fluids at the Nanoscale: From Continuum to Subcontinuum Transport. Annu. Rev. Fluid Mech. 2021, 53, 377-410.

(3) Gravelle, S.; Joly, L.; Detcheverry, F.; Ybert, C.; CottinBizonne, C.; Bocquet, L. Optimizing water permeability through the hourglass shape of aquaporins. Proc. Natl. Acad. Sci. U.S.A. 2013, 110, 16367-16372.

(4) Jungwirth, P. Biological Water or Rather Water in Biology? J. Phys. Chem. Lett. 2015, 6, 2449-2451.

(5) Knight, A. W.; Kalugin, N. G.; Coker, E.; Ilgen, A. G. Water properties under nano-scale confinement. Sci. Rep. 2019, 9, 1-12.

(6) Sendner, C.; Horinek, D.; Bocquet, L.; Netz, R. R. Interfacial water at hydrophobic and hydrophilic surfaces: Slip, viscosity, and diffusion. Langmuir 2009, 25, 10768 10781.

(7) Kolesnikov, A. I.; Zanotti, J.-M.; Loong, C.-K.; Thiyagarajan, P.; Moravsky, A. P.; Loutfy, R. O.; Burnham, C. J. Anomalously Soft Dynamics of Water in a Nanotube: A Revelation of Nanoscale Confinement. Phys. Rev. Lett. 2004, 93, 035503.

(8) Chen, M.; Coasne, B.; Guyer, R.; Derome, D.; Carmeliet, J. Role of Hydrogen Bonding in Hysteresis Observed in Sorption-Induced Swelling of Soft Nanoporous Polymers. Nat. Commun. 2018, 9, 3507.

(9) Bocquet, L.; Charlaix, E. Nanofluidics, from bulk to interfaces. Chem. Soc. Rev. 2010, 39, 1073-1095.

(10) Siboulet, B.; Coasne, B.; Dufreche, J.-F.; Turq, P. Hydrophobic Transition in Porous Amorphous Silica. J. Phys. Chem. B 2011, 115, 7881.

(11) Zhang, Z.; Li, S.; Mi, B.; Wang, J.; Ding, J. Surface slip on rotating graphene membrane enables the temporal selectivity that breaks the permeability-selectivity tradeoff. Sci. Adv. 2020, 6, 1-8.

(12) Bellissent-Funel, M.-C. Status of experiments probing the dynamics of water in confinement. Eur. Phys. J. E 2003, 12, 83-92.

(13) Siboulet, B.; Molina, J.; Coasne, B.; Turq, P.; Dufreche, J.F. Water self-diffusion at the surface of silica glasses: effect of hydrophilic to hydrophobic transition. Mol. Phys. 2013, 111, 3410-3417.

(14) Romero-Vargas Castrillón, S.; Giovambattista, N.; Aksay, I. A.; Debenedetti, P. G. Evolution from surfaceinfluenced to bulk-like dynamics in nanoscopically confined water. J. Phys. Chem. B 2009, 113, 7973-7976.

(15) Osti, N. C.; Cote, A.; Mamontov, E.; Ramirez-Cuesta, A.; Wesolowski, D.; Diallo, S. Characteristic features of water dynamics in restricted geometries investigated with quasi-elastic neutron scattering. Chem. Phys. 2016, 465, $1-8$. 
(16) Baum, M.; Rieutord, F.; Juranyi, F.; Rey, C.; Rebiscoul, D. Dynamical and Structural Properties of Water in Silica Nanoconfinement: Impact of Pore Size, Ion Nature, and Electrolyte Concentration. Langmuir 2019, 35, 1078010794.

(17) Hummer, G.; Rasaiah, J. C.; Noworyta, J. P. Water conduction through the hydrophobic channel of a carbon nanotube. Nature 2001, 414, 188-190.

(18) Falk, K.; Sedlmeier, F.; Joly, L.; Netz, R. R.; Bocquet, L. Molecular origin of fast water transport in carbon nanotube membranes: superlubricity versus curvature dependent friction. Nano Lett. 2010, 10, 4067-4073.

(19) Jensen, M. Ø.; Mouritsen, O. G.; Peters, G. H. The hydrophobic effect: Molecular dynamics simulations of water confined between extended hydrophobic and hydrophilic surfaces. J. Chem. Phys. 2004, 120, 9729-9744.

(20) Fraux, G.; Coudert, F.-X.; Boutin, A.; Fuchs, A. H. Forced intrusion of water and aqueous solutions in microporous materials: from fundamental thermodynamics to energy storage devices. Chem. Soc. Rev. 2017, 46, 7421-7437.

(21) Chen, B.; Yang, Z.; Zhu, Y.; Xia, Y. Zeolitic imidazolate framework materials: recent progress in synthesis and applications. J. Mater. Chem. A 2014, 2, 16811-16831.

(22) Ortiz, A. U.; Freitas, A. P.; Boutin, A.; Fuchs, A. H.; Coudert, F.-X. What makes zeolitic imidazolate frameworks hydrophobic or hydrophilic? The impact of geometry and functionalization on water adsorption. Phys. Chem. Chem. Phys. 2014, 16, 9940-9949.

(23) Grosu, Y.; Gomes, S.; Renaudin, G.; Grolier, J.-P. E.; Eroshenko, V.; Nedelec, J.-M. Stability of zeolitic imidazolate frameworks: effect of forced water intrusion and framework flexibility dynamics. RSC Adv. 2015, 5, 89498-89502.

(24) Michelin-Jamois, M.; Picard, C.; Vigier, G.; Charlaix, E. Giant osmotic pressure in the forced wetting of hydrophobic nanopores. Phys. Rev. Lett. 2015, 115, 036101.

(25) Troyano, J.; Carné-Sanchez, A.; Avci, C.; Imaz, I.; Maspoch, D. Colloidal metal organic framework particles : the pioneering case of ZIF-8. Chem. Soc. Rev. 2019, $48,5534-5546$.

(26) Calero, S.; Gómez-Álvarez, P. Underlying adsorption mechanisms of water in hydrophobic and hydrophilic zeolite imidazolate frameworks: ZIF-71 and ZIF-90. J. Phys. Chem. C 2015, 119, 23774-23780.

(27) Nalaparaju, A.; Zhao, X.; Jiang, a. J. Molecular understanding for the adsorption of water and alcohols in hydrophilic and hydrophobic zeolitic metal- organic frameworks. J. Phys. Chem. C 2010, 114, 11542-11550.

(28) Fraux, G.; Boutin, A.; Fuchs, A. H.; Coudert, F.-X. Structure, Dynamics, and Thermodynamics of Intruded Electrolytes in ZIF-8. J. Phys. Chem. C 2019, 123, 15589-15598.
(29) Lowe, A.; Tsyrin, N.; Chorążewski, M.; Zajdel, P.; Mierzwa, M.; Leão, J. B.; Bleuel, M.; Feng, T.; Luo, D.; Li, M. et al. Effect of Flexibility and Nanotriboelectrification on the Dynamic Reversibility of Water Intrusion into Nanopores: Pressure-Transmitting Fluid with Frequency-Dependent Dissipation Capability. ACS Appl. Mater. Interfaces 2019, 11, 40842-40849.

(30) Han, C.; Verploegh, R. J.; Sholl, D. S. Assessing the Impact of Point Defects on Molecular Diffusion in ZIF-8 Using Molecular Simulations. J. Phys. Chem. Lett. 2018, 9, 4037-4044

(31) Amrouche, H.; Aguado, S.; Pérez-Pellitero, J.; Chizallet, C.; Siperstein, F.; Farrusseng, D.; Bats, N.; NietoDraghi, C. Experimental and computational study of functionality impact on sodalite-zeolitic imidazolate frameworks for CO2 separation. J. Phys. Chem. C 2011, $115,16425-16432$.

(32) Wolanin, J.; Giraud, J.; Payre, C.; Benoit, M.; Antonelli, C.; Quemener, D.; Tahiri, I.; Vandamme, M.; Zanotti, J.-M.; Plazanet, M. Oedometric-like setup for the study of water transport in porous media by quasielastic neutron scattering. Rev. Sci. 2021, 92, 024106.

(33) Bée, M. Quasielastic neutron scattering; Bristol, England ; Philadelphia : Adam Hilger, (C)1988.

(34) Ortiz, A. U.; Boutin, A.; Fuchs, A. H.; Coudert, F. X. Anisotropic elastic properties of flexible metal-organic frameworks: How soft are soft porous crystals? Phys. Rev. Lett. 2012, 109, 1-5.

(35) Tortora, M.; Zajdel, P.; Lowe, A. R.; Jensen, G. V.; Bleuel, M.; Giacomello, A.; Casciola, C. M.; Meloni, S.; Grosu, Y. Giant Negative Compressibility by Liquid Intrusion into Superhydrophobic Flexible Nanoporous Frameworks . Nano Lett. 2021, 21, 2848-2853.

(36) Tsimpanogiannis, I. N.; Moultos, O. A.; Franco, L. F. M.; Spera, M. B. d. M.; Erds, M.; Economou, I. G. Selfdiffusion coefficient of bulk and confined water: a critical review of classical molecular simulation studies. Mol. Simul. 2019, 45, 425-453.

(37) Qvist, J.; Schober, H.; Halle, B. Structural dynamics of supercooled water from quasielastic neutron scattering and molecular simulations. J. Chem. Phys. 2011, 134, 144508 .

(38) Bousige, C.; Levitz, P.; Coasne, B. Bridging scales in disordered porous media by mapping molecular dynamics onto intermittent Brownian motion. Nat. Commun. 2021, $12,1043$.

(39) Coasne, B.; Fourkas, J. T. Structure and dynamics of benzene confined in silica nanopores. J. Phys. Chem. C 2011, $115,15471-15479$. 
TOC Graphic

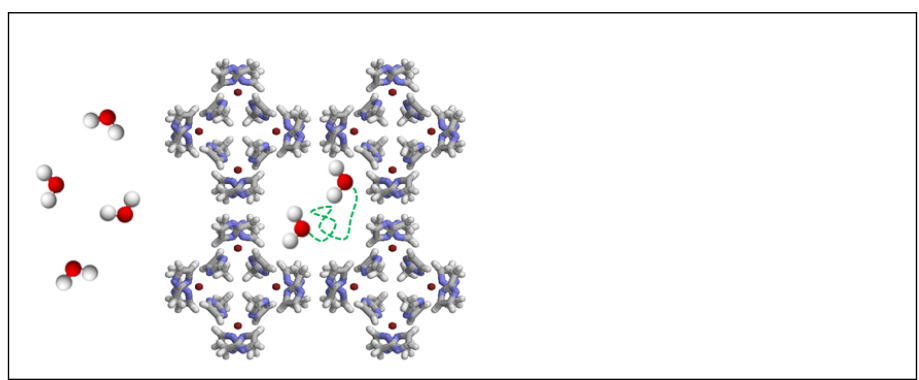

640 